\title{
AVALIAÇÃO DE PROCESSO E RESULTADO EM PSICOTERAPIAS: UMA REVISÃO
}

\author{
Ana Carolina Peuker \\ Luísa Fernanda Habigzang \\ Silvia Helena Koller" \\ Lisiane Bizarro Araujo ${ }^{\infty}$
}

\begin{abstract}
RESUMO. A avaliação em psicoterapia constitui um desafio para pesquisadores e clínicos. Esta revisão crítica sobre possibilidades de avaliação de processo e resultado em psicoterapia estimula o debate sobre a incorporação da prática clínica baseada em evidências como meio de obter informação científica quanto à adequação da intervenção terapêutica. Através de estudos sobre a avaliação do processo psicoterápico é possível elucidar conexões entre o tratamento psicológico e seus efeitos. Desta forma, torna-se possível identificar mecanismos de ação terapêutica e estratégias que podem potencializar o processo de mudança. Este debate poderá favorecer a aproximação entre pesquisa e prática clínica. Identificar variáveis das quais pode depender a eficácia/efetividade do tratamento psicoterápico repercute no aprimoramento do treinamento de psicólogos em formação e no delineamento de intervenções custo-efetivas.
\end{abstract}

Palavras-chave: Tratamento psicoterápico, eficácia e efetividade, prática baseada em evidência.

\section{ASSESSMENT OF PSYCHOTHERAPY PROCESS AND OUTCOME: A REVIEW}

\begin{abstract}
Assessment of psychotherapy is a challenge to both researchers and clinical psychologists. This critical review about assessment of process and outcomes in psychotherapy stimulates the debate on how evidence-based clinical practice can provide scientific information about the relationship between therapeutic intervention and clinical outcome. This allows to identify therapeutic mechanisms and strategies to enhance the process of change in psychotherapy. This debate might narrow the gap between research and clinical practice. The improvement on clinical psychology training and the choice of costeffective interventions might depend on the identification of variables that influence efficacy and effectiveness of psychotherapy.
\end{abstract}

Key words: psychotherapeutic treatment, efficacy and effectiveness, evidence-based practice.

\section{EVALUACIÓN DEL PROCESO Y RESULTADO EN PSICOTERAPIAS: UNA REVISIÓN}

RESUMEN. La evaluación en psicoterapia constituye un desafío para investigadores y clínicos. Esta revisión crítica sobre posibilidades de evaluación de proceso y resultado en psicoterapia estimula la discusión sobre la incorporación de la práctica clínica basada en evidencia como medio de obtener información científica para la adecuación de la intervención terapéutica. Con base en estudios sobre evaluación del proceso psicoterápico es posible aclarar conecciones entre el tratamiento psicológico y sus efectos. De esta forma, se hace posible identificar mecanismos de acción terapéutica y estrategias que pueden potencializar el proceso de mudanza. Esta discusión podrá favorecer la aproximación entre la investigación y práctica clínica. Identificar variables de las cuales puede depender la eficácia/efectividad del tratamiento psicoterápico repercute en el aprimoramiento del entrenamiento de psicólogos en formación y en el delineamiento de intervenciones custoefectiva.

Palabras-clave: Tratamiento psicoterápico, eficacia y efectividad, práctica basada en evidencia..

* Mestrado em Psicologia do Desenvolvimento pela Universidade Federal do Rio Grande do Sul, Brasil. Professor Convidado da Universidade Federal do Rio Grande do Sul , Brasil..

\# Mestrado em Psicologia do Desenvolvimento pela Universidade Federal do Rio Grande do Sul, Brasil.

II Doutorado em Educação pela Pontifícia Universidade Católica do Rio Grande do Sul, Brasil. Professor Associado II da Universidade Federal do Rio Grande do Sul , Brasil.

x Doutorado em Psicologia pelo Institute of Psychiatry King's College London, Grã-Bretanha. Professor Adjunto II da Universidade Federal do Rio Grande do Sul , Brasil. 


\section{AVALIAÇÃO DE PROCESSO E RESULTADO EM PSICOTERAPIAS}

A avaliação do processo psicoterápico e o conseqüente efeito das intervenções psicológicas tem sido um desafio para pesquisadores e clínicos. A articulação de conhecimentos teóricos, técnicos, metodológicos e éticos é fundamental para avaliar o processo terapêutico e verificar seu efeito. Embora exista consenso na literatura quanto aos benefícios gerados pela psicoterapia (Roth \& Fonagy, 2005), ainda se faz necessário conhecer em profundidade quais aspectos desta prática favorecem resultados positivos. Isto se dá, em especial, no cenário contemporâneo, no qual implicações sociais, políticas e econômicas exigem a demonstração concreta de que os tratamentos em saúde mental são efetivos e necessários. Os objetivos do presente artigo são: apresentar e discutir possibilidades de avaliação do processo e dos resultados da psicoterapia; e debater sobre a incorporação da prática clínica baseada em evidências como uma alternativa para obter informação científica quanto à adequação da intervenção psicológica.

Os avanços mais significativos no âmbito da pesquisa em psicoterapia estão direcionados à compreensão do contexto das interações terapêuticas através de métodos que creditem cientificidade ao conhecimento produzido no campo. Para tanto, é necessário que sejam conhecidos em profundidade os fatores que podem estar relacionados ao processoresultado da psicoterapia.

\section{VARIÁVEIS ASSOCIADAS AO PROCESSO- RESULTADO DA PSICOTERAPIA}

A psicoterapia pode resultar em benefícios como redução de problemas sociais, emocionais e comportamentais (Ex.: ideação suicida, depressão e pânico), alteração de condições físicas (Ex.: dor, pressão arterial), potencialização do processo de recuperação de cirurgias ou doenças e melhora na qualidade de vida (Roth \& Fonagy, 2005). O efeito de uma intervenção psicoterápica pode ocorrer pela interação de variáveis relacionadas ao paciente, ao terapeuta e à relação estabelecida entre eles (aspecto interpessoal do processo).

Considerando as variáveis relacionadas ao paciente, pode-se destacar a natureza do transtorno que ele é portador, sua história de vida e clínica pregressa, a presença de rede de apoio social e afetiva e a motivação para o processo de mudança (Cordioli, 2003; Habigzang \& Koller, 2006). Dentre os aspectos associados ao quadro psicopatológico, que podem repercutir no processo e no resultado da intervenção, destacam-se: severidade e duração da doença, prejuízos de ordem cognitiva, déficits comportamentais graves, problemas interpessoais, familiares e conjugais. Além disso, as co-morbidades, como uso de substâncias psicoativas e transtornos de personalidade, também podem configurar-se como dificuldades para o sucesso do tratamento. De forma oposta, o comprometimento do paciente com a mudança, a confiança nos procedimentos e resultados da psicoterapia, podem contribuir para o sucesso terapêutico (Ito, 2001).

As variáveis relacionadas ao terapeuta, que podem repercutir tanto no processo como na resposta à intervenção psicoterápica, são: a sua competência técnica, a sua experiência clínica e seu estilo pessoal. $\mathrm{O}$ estilo pessoal do terapeuta compreende características, tais como autenticidade, capacidade empática, entre outras. Tais variáveis contribuem para o desenvolvimento de hipóteses diagnósticas e da avaliação detalhada da queixa e dos problemas vivenciados pelo paciente. Além disso, essas variáveis possibilitam o escrutínio dos fatores que representam obstáculos e/ou facilitadores ao tratamento (Ito, 2001).

Dessa forma, a conceituação precisa do caso possibilita o planejamento adequado do processo de intervenção. O plano de tratamento deve incluir os aspectos individuais do paciente, as características do quadro psicopatológico apresentado por ele e a utilização de técnicas efetivas (Araújo \& Shinohara, 2002; Caminha \& Habigzang, 2003). A aplicação correta das técnicas indicadas para o tratamento também é importante. A definição destas técnicas pode ser dar a partir de estudos clínicos que avaliam sua efetividade ou eficácia para cada tipo de transtorno psicológico (David, 2004). Estes aspectos relativos ao terapeuta, podem ser estimulados e desenvolvidos no decorrer da sua formação e assim, aumentar os índices de melhora dos pacientes através do estabelecimento de uma boa relação terapêutica. A perícia de um terapeuta está vinculada a sua capacidade de estabelecer um contexto propício para a realização de intervenções efetivas e para a troca de feedbacks (Oliveira, Nunes, Fernández-Álvarez, \& Garcia, 2006).

Além das variáveis específicas associadas ao paciente e ao terapeuta, o resultado de uma intervenção psicoterápica também dependerá da relação de trabalho estabelecida entre eles, a aliança terapêutica (AT). O termo AT compreende o vínculo de confiança entre terapeuta e paciente, bem como a capacidade da dupla para realizar as tarefas 
psicoterápicas (Cordioli, 2003; Safran, 2002). Enquanto a definição de natureza e de função da aliança terapêutica vem sendo desenvolvida com o passar dos anos, sua importância no processo de mudança é indiscutível. A AT tem sido considerada um importante preditor de resultados terapêuticos em diferentes abordagens psicoterápicas (Marcolino \& Iacoponi, 2001; Oliveira et al., 2006; Serralta, Nunes \& Eizirik, 2007).

Focalizar a pesquisa no estabelecimento da aliança terapêutica e reparação de rupturas na aliança pode constituir uma linha de investigação promissora. Tais investigações podem ser úteis para melhorar a efetividade e a eficácia da psicoterapia e reduzir as taxas de abandono. Pois, a ruptura da AT pode desempenhar papel decisivo no abandono da terapia. Neste sentido, a detalhada análise das interações paciente-terapeuta pode prover conhecimentos específicos sobre o estabelecimento e a reparação de rupturas na aliança terapêutica (Muran, Segal, Samstag, \& Crawford, 1994; Oliveira et al., 2006).

De forma geral, investigações sobre efetividade, eficácia, tratamentos focalizados e avaliações que combinaram efetividade e eficácia confirmam os efeitos positivos da psicoterapia, culminando no reconhecimento de que muitos tratamentos têm fortes evidências a seu favor. Apesar disso, depois de décadas de pesquisa em psicoterapia, ainda não há uma explicação baseada em evidência de como ou porque a maior parte das intervenções produz mudança, isto é, o que é importante para que um tratamento funcione (Kazdin, 2007). Conhecer como estes efeitos se dão pode elucidar conexões entre o que é feito (tratamento) e os diversos resultados, permitindo direcionar estratégias que podem desencadear o processo de mudança em psicoterapia.

\section{A INVESTIGAÇÃO DO PROCESSO DE MUDANÇA EM PSICOTERAPIA}

Através de estudos sobre o processo terapêutico é possível compreender como se dá a mudança no decorrer do tratamento e identificar os mecanismos de ação terapêutica. Para isso, podem ser empregados métodos qualitativos e quantitativos para investigar padrões de relacionamento e comunicação entre a dupla terapeuta/paciente ao longo das sessões de terapia, fazendo relações com eventos terapêuticos positivos e negativos, assim como a mudança clínica (Serralta, Nunes \& Eizirik, 2007). Além disso, compreender como o trabalho terapêutico ocorre pode ajudar a identificar variáveis das quais pode depender a eficácia de um dado tratamento. Por exemplo, se mudanças no processo cognitivo contribuem para a mudança terapêutica, o status pré-tratamento dos processos relacionados devem ser considerados (p.ex. raciocínio abstrato, resolução de problemas), uma vez que, tais processos, podem moderar quem responde ou falha em responder ao tratamento (Kazdin, 2007).

A demonstração de uma forte associação entre a intervenção psicoterápica (A) e um mediador de mudança hipotético (B) é um requisito primordial para que se conheça o mecanismo que precipita a mudança terapêutica (C). Por exemplo, um ensaio clínico randomizado (ECR) pode demonstrar que um determinado tratamento quando comparado ao placebo leva à mudança. Isto é, pode-se dizer que o tratamento causou a mudança. Mas, demonstrar a causa não significa dizer por que a intervenção ocasionou a mudança ou como a mudança ocorreu. Para tal, é necessário que se evidencie uma forte associação entre as variáveis A, B e C. Do contrário, o mediador de mudança hipotético se torna enfraquecido, passível de ser eliminado (Kazdin, 2007).

A demonstração de que a associação encontrada entre intervenção, mediador proposto e resultado é específica se atende ao critério da especificidade. $\mathrm{Ou}$ seja, deve-se buscar identificar conexões específicas mais do que múltiplos mediadores que contribuem para a mudança terapêutica. Caso seja demonstrado que muitos construtos plausíveis não interferem na mudança terapêutica, com exceção de um, a hipótese de que este último media a mudança é fortalecido (Kazdin, 2007).

A identificação de variáveis das quais pode depender a eficácia/efetividade de um tratamento pode contribuir para o delineamento de estratégias úteis para a obtenção da mudança terapêutica. Desta maneira, estudos experimentais podem favorecer a construção de evidências científicas que subsidiarão a prática psicoterapêutica.

\section{CONSTRUÇÃO DE EVIDÊNCIAS NA AVALIAÇÃO DE PROCESSO-RESULTADOS}

Os estudos experimentais, como os ensaios clínicos randomizados, constituem uma ferramenta essencial na construção de evidências científicas para a prática clínica (Coutinho \& Cunha, 2005; Coutinho, Huf, \& Bloch, 2003). Embora os termos eficácia e efetividade sejam utilizados como sinônimos, estes apresentam diferenças significativas quanto aos objetivos e aos métodos dos ensaios clínicos (Silva \& Formigli, 1994).

Os estudos de eficácia têm como objetivo avaliar a existência de uma relação causal entre o tratamento e 
a resposta. O foco está na validade interna e, em uma avaliação modelo, as amostras se caracterizam pela homogeneidade, com critérios de inclusão e exclusão altamente definidos, retirando, por exemplo, os participantes que apresentam co-morbidades. O delineamento deve ser experimental com alocação aleatória dos participantes nos grupos experimental e controle. O setting terapêutico precisa ser controlado e a intervenção claramente definida, baseada em manual, seguindo protocolos padronizados. Os clínicos são rigorosamente treinados, monitorados e supervisionados (Nash, McCrory, Nicholson, \& Andrasik, 2005).

Os estudos de efetividade objetivam avaliar a resposta ao tratamento em um setting semelhante ao "mundo real". O foco está na validade externa e as amostras são heterogêneas, incluindo participantes que apresentam comorbidades típicas. O delineamento destes estudos é quase-experimental, incluindo a randomização dos participantes. A intervenção visa à adaptação ao setting natural, sendo que o setting de pesquisa é menos controlado e representativo da prática clínica. Os clínicos possuem níveis variados de treinamento, monitoramento e supervisão (Nash et al., 2005).

Considerando as distinções apontadas, pode-se concluir que os estudos de eficácia contribuem para o estabelecimento de relação causal entre tratamento e resultado. Contudo, ensaios clínicos que visam à verificação da eficácia do tratamento são metodologicamente mais difíceis de serem realizados e apresentam maiores custos.

De acordo com o exposto, é importante verificar em que medida os tratamentos que demonstraram ser bem-sucedidos na redução de sintomas, dito eficazes, apresentam significância clínica (Kendall, 1999). No cerne da discussão pela a busca da eficácia, os ensaios clínicos controlados geram controvérsias entre os pesquisadores que os defendem (e privilegiam a evidência científica) e aqueles que o criticam. Estes últimos $\mathrm{o}$ fazem por não encontrar sentido em resultados oriundos de um contexto artificial, altamente controlado e, portanto, sem utilidade clínica. Neste ponto, emerge a discussão sobre a significância clínica e a significância estatística, a segunda priorizada pelas terapias sustentadas empiricamente (Enéas, 2007).

Os estudos de efetividade apresentam como vantagem a generalização dos resultados, uma vez que estes buscam reproduzir ao máximo as condições de atendimento encontradas na rotina dos psicoterapeutas, tendo maior utilidade no campo da clínica. Os ensaios clínicos de eficácia e de efetividade não devem ser considerados antagônicos, mas complementares na produção de evidências sobre intervenções em saúde (Coutinho, Huf, \& Bloch, 2003).

Outro aspecto importante sobre os ensaios clínicos é que estes baseiam sua avaliação na comparação de resultados do pré e pós-testes, que se constituem em medidas quantitativas de sintomas psicológicos. Contudo, o resultado da intervenção não ocorre apenas no final do tratamento. Evidências de sucesso terapêutico começam a se acumular já no início da terapia, uma vez que mudanças terapêuticas significativas podem estar concentradas em sessões críticas específicas (Dobson, 2001). Portanto, no contexto de pesquisa, os delineamentos que analisam a correlação de variáveis em uma amostra aleatória de sessões podem ser inadequados, pois eventos cruciais para a evolução do tratamento podem ser omitidos da análise.

Os métodos de pesquisa aplicados à psicoterapia tornaram-se mais objetivos, padronizados e rigorosos a partir da década de 1950. Isto gerou a expectativa de que fatos empíricos e observações objetivas ocupariam o lugar de especulações como base para os avanços na área. Contudo, observa-se ainda hoje uma distância entre as constatações científicas e a prática psicoterápica (Dobson, 2001; Goldfried \& Wolfe, 1996).

\section{DISTÂNCIA ENTRE PESQUISA - PRÁTICA}

Embora a pesquisa de resultados forneça dados com importantes implicações para a prática clínica, ainda há uma defasagem na incorporação destas informações científicas como base para delinear ou atualizar a prática psicoterápica. A desconsideração dos achados científicos pode implicar em sérias questões éticas (Dobson, 2001; Goldfried \& Wolfe, 1996; Williams, Beutler, Wakefield, \& Entwistle, 1995).

Em geral, há uma distância entre a natureza global dos achados de pesquisa e a natureza específica dos dilemas clínicos. Muitas vezes, os dados obtidos através de ensaios clínicos sobre eficácia também não oferecem alternativas de como resolver impasses e dilemas clínicos característicos da psicoterapia, independente de sua natureza específica ou do transtorno que está sendo tratado. Apesar disso, tentativas têm sido feitas para traduzir os achados de pesquisa em recomendações para a prática clínica, com o objetivo de sustentar intervenções terapêuticas em uma base empírica confiável (Por exemplo, 
Bernardo, Nobre, \& Jatene, 2004; Goldfried \& Wolfe, 1996; Lima, Soares, \& Bacaltchuk, 2000).

O paradigma de pesquisa adotado atualmente em psicoterapia deve ser ampliado para que se dissipe a distância entre pesquisa e prática psicoterápica. Este novo paradigma de pesquisa em psicoterapia deve elucidar os diversos processos de mudança e individualizar as intervenções em investigação. Além disso, a colaboração entre clínicos e pesquisadores deve ser otimizada (Goldfried \& Wolfe, 1996; Hoshman \& Polkinghorne, 1992; Peterson, 1991; Piccinini, 1996; Stricker, 1992).

Terapias padronizadas que foram testadas e revelaram eficácia necessitam ser aplicadas em mais de um local, tais como centros de atenção primária, clínicas ambulatoriais e centros comunitários de saúde mental. Dessa forma, estes estudos poderiam fornecer informações mais precisas sobre os efeitos do tratamento, uma vez que seriam aplicados em contextos distintos, mais próximos da vida real. Eventos vitais, características de personalidade, assim como outras variáveis que possam estar implicadas na etiologia ou manutenção dos sintomas referentes ao transtorno em estudo devem ser investigados através de ensaios clínicos sobre efetividade (Goldfried \& Wolfe, 1996).

A distância entre a pesquisa em psicoterapia e a prática clínica pode ser superada se estas áreas forem compreendidas como complementares desde a formação do psicoterapeuta. Os cursos de formação em psicoterapia podem contribuir para a integração da pesquisa e da prática, articulando-as em seus programas de ensino. A pesquisa e a prática têm uma mútua dependência no conjunto do conhecimento em psicoterapia (Hoshman \& Polinghorne, 1992; Peterson, 1991; Piccinini, 1996; Stricker, 1992).

Geralmente, os psicoterapeutas necessitam tomar decisões complexas que incluem a escolha do tratamento mais adequado, considerando a efetividade, tolerabilidade e a relação custo-benefício de sua escolha. A complexidade dessa escolha remete à avaliação crítica dos resultados de pesquisas sistemáticas, baseadas em cuidadosa revisão de artigos científicos (Bernardo, Nobre, \& Jatene, 2004; Lima, Soares, \& Bacaltchuk, 2000).

É importante que o trabalho psicoterápico esteja associado à pesquisa e seja fundamentado em uma teoria pautada por evidências científicas. Contudo, isto não significa que esta prática orientada limite a forma como a psicoterapia é conduzida e seja ditada somente por resultados empíricos. De forma similar, é igualmente necessário, que as conclusões sobre o efeito terapêutico não se distanciem da realidade clínica. Ou seja, a integração entre teoria-pesquisa deve ser estabelecida (Goldfried \& Wolfe, 1996; Hoshman \& Polkinghorne, 1992; Peterson, 1991; Piccinini, 1996; Stricker, 1992).

\section{O USO DE ESCALAS E INSTRUMENTOS PSICOLÓGICOS NO CONTEXTO DA AVALIAÇÃO DE PROCESSO-RESULTADO}

O uso de instrumentos padronizados pode contribuir de muitas maneiras para a avaliação de intervenções e tratamentos e, assim, auxiliar na identificação das variáveis que podem estar atreladas ao sucesso e/ou fracasso do tratamento psicoterápico.

No que concerne à pesquisa científica, o emprego de instrumentos de medida garante que a coleta de dados seja realizada de forma padronizada. Assim, informações acerca de sintomas específicos (em geral, freqüência e intensidade) podem ser comparadas entre indivíduos de forma fidedigna (Ito \& Ramos, 1998). Registros objetivos do processo terapêutico (por exemplo, uso de áudio ou de vídeo) favorecem uma compreensão mais precisa do que ocorreu na sessão. Além disso, podem ser estudados, exaustivamente, pelo terapeuta ou por juízes independentes. Quando estes últimos realizam as avaliações, deve-se utilizar categorias de análise operacionalmente definidas a fim de se obter bons índices de precisão dos julgamentos efetuados. Assim, torna-se possível identificar fatores que tenham valor preditivo e que possam ser generalizados (Greenberg \& Newman, 1996).

A utilização de medidas que avaliam a intensidade e freqüência dos sintomas, bem como a avaliação qualitativa do engajamento do paciente no processo de mudança fornecem estimativas acerca do impacto da intervenção (Yoshida, 1998). Além disso, o procedimento de incluir em uma mesma investigação medidas oriundas de fontes distintas (triangulação) também compõe as orientações metodológicas referentes às pesquisas em psicoterapia.

Através da triangulação medidas fornecidas pelo terapeuta, por juízes independentes e pelo paciente podem ser comparadas, complementando-se, confirmando-se ou mesmo contrapondo-se. Medidas fornecidas pelos próprios pacientes, através de instrumentos de avaliação auto-administrados (selfreport), permitem investigar vários aspectos do processo psicoterápico. Entre eles, a percepção do sujeito quanto: à remissão do sintoma, ao grau de satisfação obtido com o processo, ou à adequação da ajuda prestada pelo terapeuta em relação ao motivo da consulta (Yoshida, 1998). 
Já no âmbito da prática clínica, o uso de escalas e de instrumentos psicológicos pode contribuir na avaliação da evolução clínica do paciente antes, durante e ao término da intervenção. Uma revisão de literatura sobre os ensaios clínicos pode orientar a escolha de técnicas mais adequadas para o problema apresentado pelo paciente, tendo como base a conceituação do caso. A aplicação de medidas repetidas que avaliem a intensidade e freqüência dos sintomas, bem como a avaliação qualitativa do engajamento do paciente no processo de mudança podem contribuir para que o clínico tenha conhecimento do impacto da intervenção.

$\mathrm{O}$ uso de escalas permite que as informações colhidas na avaliação inicial sejam transformadas em um escore numérico que indica a freqüência e a gravidade dos sintomas. Essa informação também pode ser relevante na escolha do tipo de tratamento mais adequado, dependendo da característica da sintomatologia. Pode-se repetir a avaliação em mais de um momento para avaliar a resposta terapêutica. Esta reavaliação através de escalas fornece dados objetivos a respeito da evolução do paciente. Além disso, ao discutir com o paciente sobre suas respostas às escalas torna-se viável analisar objetivamente seu estado clínico e o progresso do tratamento (Ito \& Ramos, 1998).

A partir desta breve revisão obervou-se que o campo da pesquisa em psicoterapia progrediu. Ademais, constatou-se que, de um modo geral, certos protocolos de tratamento psicoterápico possuem eficácia comprovada. Contudo, isso não significa dizer que a teoria como um todo é eficaz. A área ainda carece de maior precisão considerando a complexidade do objeto de estudo em análise.

\section{CONSIDERAÇÕES FINAIS}

No contexto brasileiro, as pesquisas sobre psicoterapia ainda são incipientes. Existem poucos estudos sistemáticos sobre o processo psicoterápico e as medidas disponíveis para os pesquisadores desta área são escassas. A avaliação do processo psicoterápico e o conseqüente efeito das intervenções psicológicas permanece sendo um importante desafio para pesquisadores e clínicos. Os grandes focos de discussão da área concentram-se na busca de evidência científica e a articulação entre a pesquisa e a prática clínica. A discrepância entre pesquisa e prática ainda persiste, apesar do maior rigor metodológico empregado na pesquisa em psicoterapia em relação às últimas décadas. Por isso, esforços devem ser direcionados para consolidar a colaboração entre os campos da pesquisa e da prática em psicoterapia.

Identificar variáveis das quais pode depender a eficácia/efetividade de um determinado tratamento favorece estratégias que podem servir como catalizadores do processo de mudança. Tais investigações contribuem à área de ensino, fomentam a discussão teórica-técnica e auxiliam no aprimoramento de terapeutas em formação. Além disso, geram conhecimento que favorece $o$ atendimento adequado das demandas dos pacientes e repercute no sistema público de saúde, no momento em que intervenções custo-efetivas podem ser implementadas.

$\mathrm{O}$ respeito às peculiaridades de cada caso, considerando as singularidades do paciente e do terapeuta, seus potenciais e limitações e as características do vínculo estabelecido entre eles podem refletir em maior resposta terapêutica. Portanto, variáveis associadas ao processo psicoterápico, incluindo aquelas relacionadas ao terapeuta, ao paciente e à relação terapêutica que estão implicadas no fracasso e/ou no sucesso devem continuar sendo exploradas. Neste sentido, tanto delineamentos quanti como qualitativos podem produzir dados que permitam responder às complexas questões advindas do processo terapêutico.

\section{REFERÊNCIAS}

Araújo, C. F. \& Shinohara, H. (2002). Avaliação e diagnóstico em terapia cognitivo-comportamental. Interação em Psicologia, 6(1), 37-43.

Bernardo, W. M., Nobre, M. R. C. \& Jatene, F. B. (2004). A prática clínica baseada em evidências: Buscando as evidências em fontes de informação. Revista Associação Médica Brasileira, 50(1), 104108.

Caminha, R. M. \& Habigzang, L. F. (2003). Conceituação cognitiva comportamental. Em: R. M. Caminha, R. Wainer \& N. Picolotto (Orgs.), Psicoterapias cognitivo comportamentais: teoria e prática (pp.29-37). São Paulo: Casa do Psicólogo.

Cordioli, A.(2003). Carta aos editores. Revista Brasileira de Psiquiatria, 25(1), 56-58.

Coutinho, E. S. F. \& Cunha, G. M. (2005). Conceitos básicos de epidemiologia e estatística para a leitura de ensaios clínicos controlados. Revista Brasileira de Psiquiatria, 27(2), 146-251.

Coutinho, E. S. F, Huf, G. \& Bloch, K. V. (2003). Ensaios clínicos pragmáticos: Uma opção na construção de evidências em saúde. Caderno de Saúde Pública, 19(4), 1189-1193.

David, D. (2004). Special issue on the cognitive revolution in clinical psychology: Beyond the behavioral approach - conclusions: Toward an evidence-based psychology and psychotherapy. Journal of Clinical Psychology, 60(4), 447-451. 
Dobson, K. S. (2001). Handbook of cognitive-behavioral therapies. New York: Guilford.

Enéas, M. L. E. (2007). Pesquisas em psicoterapia: sessões especiais de periódico (1995 a 2005). Psicologia: Teoria e Pesquisa, 23, 333-340.

Goldfried, M. \& Wolfe, B. E. (1996). Psychotherapy practice and research: repairing a strained alliance. American Psychologist, 51(10), 1007-1016.

Greenberg, L. S. \& Newman, F. L. (1996). An approach to psychotherapy change process research: Introduction to the special section. Journal of consulting and clinical psychology, 64(3), $435-438$.

Habigzang, L. F. \& Koller, S. H. (2006). Terapia cognitivocomportamental e promoção de resiliência para crianças e adolescentes vítimas de violência sexual intrafamiliar. Em: D. Dell'Aglio, S. Koller \& M. A. M. Yunes (Orgs.), Resiliência e psicologia positiva: interfaces do risco à proteção (pp.233-258). São Paulo: Casa do Psicólogo.

Habigzang, L. F., Hatzenberger, R., Dala Corte, F., Stroeher, F. \& Koller, S. H. (2006). Grupoterapia cognitivo-comportamental para meninas vítimas de abuso sexual: Descrição de um modelo de intervenção. Psicologia Clínica, 18(2), 163-182.

Hoshman, L. T. \& Polkinghorne, D. E. (1992). Redefining the science-practice relationship and professional training. American Psychologist, 47, 55-66.

Ito, L.M. (2001). Abordagem cognitivo-comportamental do transtorno de pânico. Revista de Psiquiatria Clínica, 28(6), 313-317.

Ito, L.M. \& Ramos, R. T. (1998). Escalas de avaliação clínica: transtorno de pânico. Revista de Psiquiatria Clínica, 25, 294-302.

Kazdin, A.E. (2007). Mediators and mechanisms of change in psychotherapy research. Annual Review of Clinical Psychology, 3, 1-27.

Kendall, P.C. (1999). Clinical significance. Journal of Consulting and Clinical Psychology, 67, 283-284.

Lima, M. S., Soares, B. G. O., \& Bacaltchuk, J. (2000). Psiquiatria baseada em evidências. Revista Brasileira de Psiquiatria, 22(3), 142-146.

Marcolino, J. A. M. \& Iacoponi, E. (2001). Escala de aliança psicoterápica da Califórnia na versão do paciente. Revista Brasileira de Psiquiatria 23(2), 88-95.
Muran, J.C., Segal, Z.V., Samstag, L.W., \& Crawford, C.E. (1994). Patient pretreatment interpersonal problems and therapeutic alliance in short-term cognitive therapy. Journal of Consulting and Clinical Psychology, 62, 185-190.

Nash, J. M., McCrory, D., Nicholson, R. A., \& Andrasik, F. (2005). Efficacy and effectiveness approaches in behavioral treatment trials. Headache, 45, 507-512.

Oliveira, M.S.; Nunes, M.L.T.; Fernández-Álvarez, H. \& Garcia, F. (2006). Estilo pessoal do terapeuta: dados preliminares da versão brasileira do EPT-Q. Psico, 37, 241-247.

Peterson, D.R. (1991). Connection and disconnection of research and pratice in the education of professional psychologist. American Psychologist, 46(4), 422-429.

Piccinini, A. (1996). Sobre o relacionamento entre pesquisador e profissional em psicologia. Em E. de M. Bonfim (Org.), Formações em Psicologia: Pós-graduação e Graduação (pp. 3140). Rio de Janeiro: Coletâneas da ANPEPP.

Roth A., \& Fonagy P. (2005). What works for whom: A critical review of psychotherapy research. London: The Guildford Press.

Safran, J. D. (2002). Ampliando os limites da terapia cognitiva: $O$ relacionamento terapêutico, a emoção e o processo de mudança. Porto Alegre: Artmed.

Serralta, F. B., Nunes, M. L. T. \& Eizirik, C. L. (2007). Elaboração da versão em portugês do Psychotherapy Process Q-Set. Revista de Psiquiatria do Rio Grande do Sul, 29(1), 44-55.

Silva, L. M. V. \& Formigli, V. L. A. (1994). Avaliação em saúde: Limites e perspectivas. Caderno de Saúde Pública, 10(1), 80-91.

Stricker, G. (1992). The relationship of research to clinical practice. American Psychologist, 47, 543-549.

Williams, R. E., Bleuter, L. E., Wakefield, P. J. \& Entwistle, S. R. (1995). Brinding scientist and practitioner perspectives in clinical psychology. American Psychologist, 50, 984-994.

Yoshida, E. M. P. (1998). Avaliação de mudança em processos terapêuticos. Psicologia Escolar e Educacional, Campinas, 2, $115-127$.

\section{Endereço para correspondência :}

Ana Carolina Peuker. Laboratório de Psicologia Experimental, Neurociências e Comportamento (LPNEc). Instituto de Psicologia (UFRGS). Rua Ramiro Barcelos, 2600, sala 2 (térreo), Bairro Santana, CEP 90035-003, Porto Alegre-RS, Brasil. E-mail: acpeuker@terra.com.br 